\title{
Promising primers for detection of phytoplasma causing coconut lethal yellowing disease in Mozambique
}

\author{
M. O. M. Mazivele • Valter Nuaila (D) - M. Durante • M. M. Colombo • E. Taviani
}

Received: 17 March 2017 / Accepted: 6 June 2018 / Published online: 27 June 2018

(C) The Author(s) 2018

\begin{abstract}
Coconut is one of the main cash crop in Mozambique, which occupied the second position after Tanzania in coconut production in Africa. Coconut production was drastically affected by the occurrence of a devastating Coconut Lethal Yellowing Disease (CLYD) epidemics, which reduced significantly the coconut yields. CLYD symptoms triggered upon phytoplasma infection, i.e. premature fruit dropping, necrosis of the inflorescence and progressive yellowing of the leaves, are used to identify infected trees. However, the diagnostic based uniquely on symptoms is not conclusive to confirm infection, and needs to be confirmed by molecular methods. In this study, three previously described reference primers for phytoplasma detection were tested on infected samples collected in Mozambique. Since those primers gave incongruent results, 20 new primer pairs targeting the $16 \mathrm{~S}$ rDNA region, were newly designed. To evaluate their performance in detecting coconut infecting phytoplasma, 108 samples were tested and selected positive samples
\end{abstract}

M. O. M. Mazivele $\cdot$ V. Nuaila $(\bowtie) \cdot$ M. M. Colombo •

E. Taviani

Center for Biotechnology, Eduardo Mondlane University, Av. de Mocambique, km 1.5, Maputo, Mozambique

e-mail: nuailavav@gmail.com

M. Durante

Department of Agricultural Plant Biology, University of Pisa, Pisa, Italy

E. Taviani

Department of Biomedical Science, University of Sassari, Sassari, Italy confirmed by sequencing. Our results showed a new primer pair more accurate and reliable compared to the reference pairs for CLYD detection in Mozambique. Moreover, the new primer pair was able to detect a new putative phytoplasma variant in Mozambique. Therefore, this study makes an important contribution to CLYD phytoplasma molecular diagnostics and its causative agent, giving insights that may be applied to the study of CLYD phytoplasma infection systems.

Keywords CLYD · Molecular diagnostic ·

Phytoplasma $\cdot$ Mozambique

\section{Introduction}

Coconut Lethal Yellowing Disease (CLYD) is a highly destructive disease associated with phytoplasmas (Weintraub and Beanland 2006). To date, the vector of CLYD phytoplasma is not yet demonstrated, however a recent study conducted in Mozambique reported the Diostrombus mkurangai, of the Derbidae family, as carrier of phytoplasma in CLYD affected areas, suggesting the possible role of the insect in the maintanance and possibly in the transmission of phytoplasma to plants (Bila et al. 2017). Beside possible transmission by means of vectors, vegetative transmission of infected plants is also significant in phytoplasma disease onset (Lee et al. 2000; Bertaccini et al. 2014). The infection can appear around at least 2 weeks after inoculation (Dickinson 2010; Bonnot et al. 2010), the symptoms 
develops between 3 to 6 months and are characterized by premature fruit dropping, necrosis of the inflorescence and progressive yellowing of the leaves starting from the basal ones (Bonnot et al. 2010).

Phytoplasmas are wall-less Gram-positive eubacteria belonging to the Mollicutes class with a low $\mathrm{G}+\mathrm{C}$ content, related to Bacillus and Clostridium spp., (Gundersen et al. 1994; Bai et al. 2006; Abdollahi et al. 2012). Currently, phytoplasmas are classified as a monophyletic group in the Acholeplasmatales order (Bai et al. 2006; Contaldo et al. 2012). These microorganisms cannot be grown artificially, suggesting that, differently from mycoplasma, their metabolism is completely dependent on the hosts (Lee et al. 2000; Kube et al. 2008). Moreover, the analysis of the $16 \mathrm{~S}$ rDNA region revealed great genetic diversity within the phytoplasmas, often reflecting geographical distribution of the strain (Lee et al. 2000; Llauger et al. 2002; Husain et al. 2002). These evidences led to define a new genus denominated "Candidatus Phytoplasma", which includes the CLYD infecting phytoplasma (Bai et al. 2006).

In Mozambique, CLYD was initially reported in 1958 in Cabo Delgado province; later in 1972 it spread to Zambezia province, and, up to the 2000 , the disease was reported with low intensity in all affected provinces (Nampula, Zambezia and Cabo Delgado) (Eden-Green 2006). At the beginning of the year 2000, CLYD reached epidemic proportions in Mozambique, with the majority of CLYD cases concentrated in Provinces located north of the Zambeze River, where a mortality rate of $100 \%$ was recorded in coconut plantations in two districts (Monjana et al. 2010; Bonnot et al. 2010). Since then, CLYD continued affecting drastically the coconut production in Mozambique with significant reduction in crop production, thus representing a major economic drawback for a country that, until 2006, was the second major coconut producer in Africa after Tanzania (EdenGreen 2006; Monjana et al. 2010).

Coconut Lethal Yellowing Disease diagnosis in Mozambique was based uniquely on symptomatology inspection, which is a somewhat complex task because the symptoms differ across varieties of coconut palms, and other biotic (Fusarium wilt) and abiotic (potassium deficiency) stressing factors induce similar symptoms, hindering an accurate and conclusive diagnosis (Monjana et al. 2010).

To overcome this scenario, a CLYD PCR based diagnostic was carried out using universal 16S rDNA primers (P1/P7) (Deng and Hiruki 1991), and two other primer sets to detect the West Africa CLYD group (' $\mathrm{Ca}$ P. palmicola'-related strains, $16 \mathrm{SrXXII-B}$, detected in Ghana, and 'Ca. P. palmicola', 16SrXXII-A, detected in Nigeria and Mozambique) (Ghana813/AKSR), and the East Africa group (Tanzania) (RhodeF/R) (Rohde et al. 1993; Tymon et al. 1998). However, after 2012, the three sets of primers began to give unreliable results for CLYD detection in Mozambican samples, suggesting that Mozambique strains may have an inadvertently genetic variability (Harrison et al. 2014).

Thus, the present study aimed at improving the PCRbased detection by designing new primers able to target efficiently and reproducibly a higher number of CLYD infections, including possible new emerging CLYD phytoplasma variants in Mozambique.

\section{Materials and methods}

Sample collection

A total of 108 coconut samples were collected in 4 coconut production provinces of Mozambique: 74 in Zambezia, a CLYD endemic area, 7 in Nampula, 8 in Cabo Delgado, where sporadic presence of CLYD was reported, and 19 samples in Inhambane, where cases of putative CLYD were recently reported.

Coconut trees sampled included those exhibiting symptoms such as necrosis of inflorescence, premature fruit drop and yellowing of leaves. Samples were marked as symptomatic if showing evident CLYD symptoms, asymptomatic if showing no evidence of CLYD symptoms but collected in areas nearby infected plants, and supposed negative if collected far from CLYD-affected areas.

Five grams of saw dust were collected from the trunk of the coconut, the second largest area of phytoplasma DNA concentration, and stored in $50 \mathrm{~mL}$ tubes containing dried silica at room temperature (Christensen et al. 2004; Oropeza et al. 2011).

\section{DNA extraction}

The DNA extraction was made according to Doyle and Doyle (1990). The extracted DNA was quantified by spectrophotometry using Nano Drop ${ }^{\mathrm{TM}} 1000$ (Thermo Scientific) and conserved at $-20^{\circ} \mathrm{C}$ until used. 
Selection of PCR controls

Five DNA samples from Zambezia and 10 from Nampula were tested by PCR using the universal primers (P1/P7), East (Rhode $\mathrm{F} / \mathrm{R}$ ) and West (G813/ASKR) Africa primer pairs. The positive amplicons were confirmed by direct sequencing and compared by BLASTn in NCBI (https://www.ncbi. nlm.nih.gov/). Samples giving sequences identical to CLYD phytoplasma strains by all three primer pairs were used as positive controls. Those samples giving sequences identical to other bacteria, were used as negative control.

Selection of candidate primers and PCR optimization

In order to find a suitable primer pair able to detect a wider range of CLYD Ca. P. strains, 20 new primer pairs were designed and named Phyto1F/R to Phyto20F/R (Table 1).

All primers were designed based on phytoplasma rDNA (5S, 16S and 23S) sequences as template using Geneious v.8.1.7 (Kearse et al. 2012), based on sequences of Coconut lethal yellowing phytoplasma isolates MZ-MF, Tanz08-01, and Awka wilt phytoplasma (Accession no. KJ528962, GU952107, Y14175, respectively).

Previously selected positive and negative ( $2 \mathrm{ng}$ ) controls were added to $15 \mu \mathrm{l}$ of Qiagen PCR Core kit (Qiagen, Germantown, USA) containing 1X buffer, $0.5 \mathrm{X}$ of Qsolution, $0.3 \mathrm{mM}$ of dNTP's, $0.3 \mu \mathrm{M}$ of primers e $0.2 \mathrm{U}$ of Taq Polimerase. The PCR reactions were performed in the Eppendorf thermocycler. After some tuning experiments, optimal parameters for Phyto1 to Phyto16 primer pairs were set: $95{ }^{\circ} \mathrm{C}$ for $3 \mathrm{~min}$, followed by $94{ }^{\circ} \mathrm{C}$ for $30 \mathrm{~s}, 59.3{ }^{\circ} \mathrm{C}$ for $30 \mathrm{~s}$, $72{ }^{\circ} \mathrm{C}$ for $55 \mathrm{~s}$, for 40 cycles and $72{ }^{\circ} \mathrm{C}$ for $10 \mathrm{~min}$. PCR conditions for Phyto 17, 18, 19 and 20 primer pairs varied only for the annealing temperature, $55{ }^{\circ} \mathrm{C}$ for the first three pairs and $57.5^{\circ} \mathrm{C}$ for the latter. All the amplicons were visualized on the TFX-20.M ultraviolet UV transluminator $230 \mathrm{~V} 50 \mathrm{~Hz}$.

Evaluation of the new candidate primers

Primers were evaluated against 30 DNA samples (Set 1) selected from a screening study of CLYD phytoplasma in endemic Mozambique regions, previosly tested by PCR and known to be positive for G813/ASKR and
RohdeF/R primers in former experiments. Those primers that were able to detect the presence of phytoplasma in all 30 Set 1 samples were selected to be tested against 33 symptomatic samples (Set 2) showing clear CLYD symptoms. Furthermore, 45 additional DNA samples, of which 38 as asymptomatic and 7 supposed negative were tested.

Sequence analysis

To confirm if the amplicons were related to CLYD phytoplasma, a BLASTn search of 17 amplicons obtained with the best performing and newly designed primers was carried out against the NCBI database (https://www.ncbi.nlm.nih.gov). Previously, raw sequences were cleaned and trimmed to eliminate poor quality reads with the CLC Genomic Workbench version 7.0.2 (https://www.qiagenbioinformatics.com) and aligned to CLYD phytoplasma reference sequences present in GenBank using ClustalW 2.0 (Larkin et al. 2007).

\section{Results}

Selection of PCR controls

Fifteen phytoplasma DNA samples were tested by PCR using primer pairs (P1/P7, G813/ASKR and RohdeF/ $\mathrm{R})$, with the following outcome: 8 samples were positive when amplified with the P1/P7 pair, 4 samples with the G813/AKSR pair and 4 samples with the RohdeF/R pair.

Out of the 8 amplicons obtained by the P1/P7 primer pair, 4 showed $98-99 \%$ sequence identity to CLYD phytoplasma strain M-182 (Accession no. EU549768), while 2 had $100 \%$ identity to CLYD phytoplasma isolate Tanz08-01, from Tanzania (Accession no. GU952107). Two (2) samples were not related to Ca. P. and showed 98-99\% sequence identity to Bacillus megaterium QM B1551 (Accession no. CP001983). Seven remaining amplicons were too short and showed no sequence identity with any sequence present in NCBI.

Two amplicon sequences obtained by the RhodeF/R primer pair showed $100 \%$ identity to the phytoplasma isolate Tanz08-01 (Accession no. GU952107), while 2 samples showed no sequence homology to any sequence in NCBI. Four amplicon sequences obtained by the G813/AKSR primer pair had 98-99\% identity 
Table 1 Candidate primers descriptions

\begin{tabular}{|c|c|c|c|}
\hline Primer region & Primer number & Sequence $\left(5^{\prime}->3^{\prime}\right)$ & Product length \\
\hline 16S rDNA & 1 & $\begin{array}{l}\text { CGCTGGCGGCGTGCCTAATA } \\
\text { CCCCACCATTACGTGCTGGCAA }\end{array}$ & 1098 \\
\hline $16 \mathrm{~S}$ rDNA & 2 & $\begin{array}{l}\text { AAGCGTGGGGAGCAAACAGG } \\
\text { TGGTTTGACGGGCGGTGTGT }\end{array}$ & 639 \\
\hline $16 \mathrm{~S}-23 \mathrm{~S}$ rDNA & 3 & $\begin{array}{l}\text { GCACGAAAGCGTGGGGAGCA } \\
\text { CCCCACCTTCCGGTAGGGAT }\end{array}$ & 763 \\
\hline $16 \mathrm{~S}-23 \mathrm{~S}$ rDNA & 4 & $\begin{array}{l}\text { GCAACGCCGCGTGAACGATG } \\
\text { CCCACCATTACGTGCTGGCAA }\end{array}$ & 735 \\
\hline 16S rDNA & 5 & $\begin{array}{l}\text { GTGCCAGCAGCCGCGGTAAT } \\
\text { CACGACACGAGCTGACGACAA }\end{array}$ & 554 \\
\hline $16 \mathrm{~S}-23 \mathrm{~S}$ rDNA & 6 & $\begin{array}{l}\text { CGAGCGCAACCCCTGTCGTT } \\
\text { TCGTGGTTTGACGGGCGGTG }\end{array}$ & 316 \\
\hline $16 \mathrm{~S}-23 \mathrm{~S}$ rDNA & 7 & $\begin{array}{l}\text { AACGAGCGCAACCCCTGTCG } \\
\text { CGTGGTTTGACGGGCGGTGT }\end{array}$ & 317 \\
\hline $16 \mathrm{~S}-23 \mathrm{~S}$ rDNA & 8 & $\begin{array}{l}\text { AACGCCGCGTGAACGACGAA } \\
\text { AACGACAGGGGTTGCGCTCG }\end{array}$ & 711 \\
\hline 16S rDNA & 9 & $\begin{array}{l}\text { GCACGAAAGCGTGGGGAGCA } \\
\text { CGACAGGGGTTGCGCTCGTT }\end{array}$ & 350 \\
\hline 16S rDNA & 10 & $\begin{array}{l}\text { ACGCTGGCGGCGTGCTTAAT } \\
\text { CGACAGGGGTTGCGCTCGTT }\end{array}$ & 1071 \\
\hline $16 \mathrm{~S}-23 \mathrm{~S}+5 \mathrm{~S}$ rDNA & 11 & $\begin{array}{l}\text { ATTAACGCTGGCGGCGTGCT } \\
\text { AACGACAGGGGTTGCGCTCG }\end{array}$ & 1077 \\
\hline $16 \mathrm{~S}-23 \mathrm{~S}+5 \mathrm{~S}$ rDNA & 12 & $\begin{array}{l}\text { TTAACGCTGGCGGCGTGCTT } \\
\text { TCGTGGTTTGACGGGCGGTG }\end{array}$ & 1369 \\
\hline $16 \mathrm{~S}-23 \mathrm{~S}$ rDNA & 13 & $\begin{array}{l}\text { GATTAACGCTGGCGGCGTGC } \\
\text { CGTGGTTTGACGGGCGGTGT }\end{array}$ & 1371 \\
\hline $16 \mathrm{~S}-23 \mathrm{~S}$ rDNA & 14 & $\begin{array}{l}\text { CGAGCAACGCCGCGTGAATG } \\
\text { GACCCAGCAAGCCGCCTACG }\end{array}$ & 348 \\
\hline $16 \mathrm{~S}-23 \mathrm{~S}$ rDNA & 15 & $\begin{array}{l}\text { CGTAGGCGGCTTGCTGGGTC } \\
\text { TCGTGGTTTGACGGGCGGTG }\end{array}$ & 683 \\
\hline $16 \mathrm{~S}-23 \mathrm{~S}$ rDNA & 16 & $\begin{array}{l}\text { GCACGAAAGCGTGGGGAGCA } \\
\text { CGTGGTTTGACGGGCGGTGT }\end{array}$ & 646 \\
\hline $16 \mathrm{~S}-23 \mathrm{~S}$ rDNA & 17 & $\begin{array}{l}\text { GCCACATTGGGACTGAGACA } \\
\text { ACCGAGTTTAACCCCGACAC }\end{array}$ & 533 \\
\hline $16 \mathrm{~S}-23 \mathrm{~S}$ rDNA & 18 & $\begin{array}{l}\text { ACAGCCACATTGGGACTGAG } \\
\text { GTTTACGGCGTGGACTACCA }\end{array}$ & 506 \\
\hline 16S-23S rDNA & 19 & $\begin{array}{l}\text { CTGACGCTGATGCACGAAAG } \\
\text { TTTGCCGAAAACTCGCGTTT }\end{array}$ & 524 \\
\hline $16 \mathrm{~S}-23 \mathrm{~S}$ rDNA & 20 & $\begin{array}{l}\text { ACGCTGGCGGCGTGCTTAAT } \\
\text { AGGAGGTGATCCATCCCCA }\end{array}$ & 1494 \\
\hline
\end{tabular}

to CLYD phytoplasma strain M-182 (Accession no. EU549768).

Based on these results, 4 samples (positive to both $\mathrm{P} 1 / \mathrm{P} 7$ and G813/AKSR) were chosen as positive controls for CLYD West Africa group ( $\mathrm{Ca}$ P. Palmicola, 16SrXXII-A), 2 samples (positive to both $\mathrm{P} 1 / \mathrm{P} 7$ and RhodeF/R) were chosen as positive controls for CLYD East Africa group and two samples were chosen as negative controls for further PCR experiments.
Selection of candidate primer pair

Eighteen samples known to be positive for the G813/AKSR primer pair and 12 samples known to be positive to the RhodeF/R primer pair in former experiments (Set 1, n=30) were tested with the newly designed 20 candidate Phyto primer pairs. According to the results, 2 primer pairs (Phyto3F/R and Phyto14F/R) were able to amplify all 30 positive samples (Table 2 ). 
Table 2 Evaluation of the ability of 2 candidate primers, Phyto3F/R and Phyto14F/R, to detect positive samples compared to RohdeF/R and G813/AKSR. Sensitivity was calculated as the number of positive detected of Set 1 and Set 2 over the total of true positives $(n=63)$

\begin{tabular}{llllll}
\hline & \multirow{2}{*}{ No. of samples } & No. of positive & & \\
\cline { 3 - 5 } & & Phyto3 F/R & Phyto14 F/R & Rhode F/R & G813/AKSR \\
\hline Set 1 & 30 & 30 & 30 & 18 & 12 \\
Set 2 & 33 & 14 & 33 & 4 & 8 \\
Asymptomatic & 38 & 7 & 20 & 0 & 8 \\
Supposed negative & 7 & 0 & 0 & 0 & 0 \\
Sensitivity (\%) & 70 & 100 & 35 & 32 \\
\hline
\end{tabular}

To evaluate detection efficiency, the Phyto3F/R and Phyto14F/R primer pairs were used to amplify 33 DNA samples obtained from symptomatic plants, showing clear CLYD symptoms, which were never tested before by PCR (Set 2) (Table 2).

Among Set 2 samples, the Phyto3F/R primer pair detected as positive 14 out of $33(42.4 \%)$ while the Phyto14F/R primer pair detected as positive 33 out of $33(100 \%)$ (Table 2).

Furthermore, 45 samples, of which 38 were considered from a clinical evaluation as asymptomatic and 7 as supposed negative were also tested. The Phyto3F/R primer pair detected as positive 7 out of $38(18.4 \%)$ of the asymptomatic, and 0 out of 7 of the negative samples while the Phyto $14 \mathrm{~F} / \mathrm{R}$ detected as positive 20 out of 38 $(52.6 \%)$ of the asymptomatic and 0 out of 7 of the negative samples (Table 2).

Results obtained with Phyto3F/R and Phyto14F/R primer pair against all samples were compared with those obtained with G813/AKSR and RohdeF/R primer pairs (Table 2). The sensitivity (number of positive detected / number of true positive) of the primers over a total of 63 true positive (30 of Set $1+33$ of Set 2) was $32 \%, 35 \%$, 70 and $100 \%$ for G813/AKSR, RohdeF/R, Phyto3F/R and Phyto14F/R primer pairs, respectively (Table 2).

Therefore, the experiments strongly supported that Phyto14F/R (Forward 5'-CGAGCAACGCCGCG TGAATG-3', Reverse 5'-GACCCAGCAAGCCG CCTACG-3', amplicon size $350 \mathrm{bp}$ ) was the best candidate to be used for the detection of CLYD phytoplasma in PCR experiments with samples from Mozambique, with a more accurate detection efficiency compared to G813/AKSR and RhodeF/R primer pairs.

To confirm the efficiency of the Phyto14F/R primer, we randomly selected 17 amplicons for sequencing, among which 10 were positive only with the Phyto14F/R pair, and the other 7 samples were also positive for Ghana813/ ASKR $(n=4)$ and RhodeF/R $(n=3)$ (Table 3$)$. The BLASTn analysis of the 17 sequences showed that all 17 amplicons were 98-100\% homologous to CLYD phytoplasma (Accession no. EF186805, EU549768 and GU952107) (Table 3). Eleven samples were homologous (93-98\% identity for $93-100 \%$ sequence length) to the East Africa group phytoplasma (Accession no. AC GU9252107.1), while 6 contained DNA showing 99\% sequence identity for $94-100 \%$ of sequence length to a phytoplasma strain isolated in Mozambique in 2014 (Accession no. AC KJ528984.1) (Bila et al. 2014). The latter were also positive with the G813/AKSR primer pair, generally detecting the West Africa group ' $\mathrm{Ca}$ Phytoplasma palmicola' $16 \mathrm{SrXXII}$ and related strains), and the RhodeF/R pair (1) specific to East Africa phytoplasma (Table 3).

The relative position of the three primer pairs was investigated. The resulting map showed that the Phyto14 pair annealed in a region of the phytoplasma $16 \mathrm{~S}$ rDNA which is more conserved compared to the G813/AKSR and RhodeF/R (Fig. 1). The alignment of 8 representative strains, including representatives strains from Mozambique, West and East Africa as well as Caribbean strains, showed a significant polymorphism within the annealing regions of the G813/AKSR and RhodeF/R pairs compared to a single nucleotide mismatch observed in the annealing region of the Phyto14R primer (Fig. 1).

\section{Discussion}

High genome variability in phytoplasma was described by Razin (2007), as resulting of repeated regions grouped in potential mobile units (PMUs) presenting 
Table 3 Origin and eventual concurrent positivity by G813/AKSR and RohdeF/R amplification of 17 amplicons generated by Phyto14 primer pair. The Accession number of each sequence is indicated. Percent of sequence identity to published reference sequences are indicated with corresponding Accession numbers: KJ528962, a CLYD sample from Mozambique and GU952107, a CLYD sample from Tanzania (East Africa group)

\begin{tabular}{|c|c|c|c|c|c|c|c|}
\hline No. & Province & G813/AKSR & RohdeF/R & Sequence accession no. & $\%$ of identity & $\%$ of coverage & Accession no. \\
\hline 3 & Cabo Delgado & - & + & Poor quality & & & \\
\hline 11 & Quelimane & + & - & MF782645 & 99 & 95 & KJ528962 \\
\hline 14 & Quelimane & - & - & MF782644 & 99 & 94 & KJ528962 \\
\hline 15 & Quelimane & + & - & MF782643 & 99 & 100 & KJ528962 \\
\hline 16 & Quelimane & + & - & MF782642 & 99 & 95 & KJ528962 \\
\hline 17 & Inhambane & + & + & MF782641 & 99 & 94 & KJ528962 \\
\hline 19 & Quelimane & - & + & Poor quality & & & \\
\hline 20 & Quelimane & - & - & MF782640 & 93 & 99 & GU952107 \\
\hline 21 & Quelimane & - & - & MF782639 & 98 & 99 & GU952107 \\
\hline 23 & Quelimane & - & - & MF782647 & 98 & 99 & GU952107 \\
\hline 24 & Quelimane & - & - & MF782646 & 94 & 98 & GU952107 \\
\hline 25 & Quelimane & - & - & MF782648 & 93 & 100 & GU952107 \\
\hline 26 & Quelimane & - & + & MF782652 & 97 & 98 & GU952107 \\
\hline 27 & Quelimane & - & - & Poor quality & & & \\
\hline 28 & Quelimane & - & - & MF782651 & 95 & 97 & GU952107 \\
\hline 30 & Quelimane & - & - & MF782650 & 97 & 97 & GU952107 \\
\hline 31 & Quelimane & - & - & MF782649 & 97 & 98 & GU952107 \\
\hline
\end{tabular}

numerous transposases and insertion sequences. Evidence of high variability of $16 \mathrm{~S}$ rDNA regions in phytoplasma was also evidenced by RFLP analyses (Harrison et al. 2014). It is not surprising then, that the

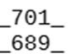

Tanzania

Mozambique

Cote d'Ivoire

Mozambique

Tanzania

Florida

Cuba

Mozambique
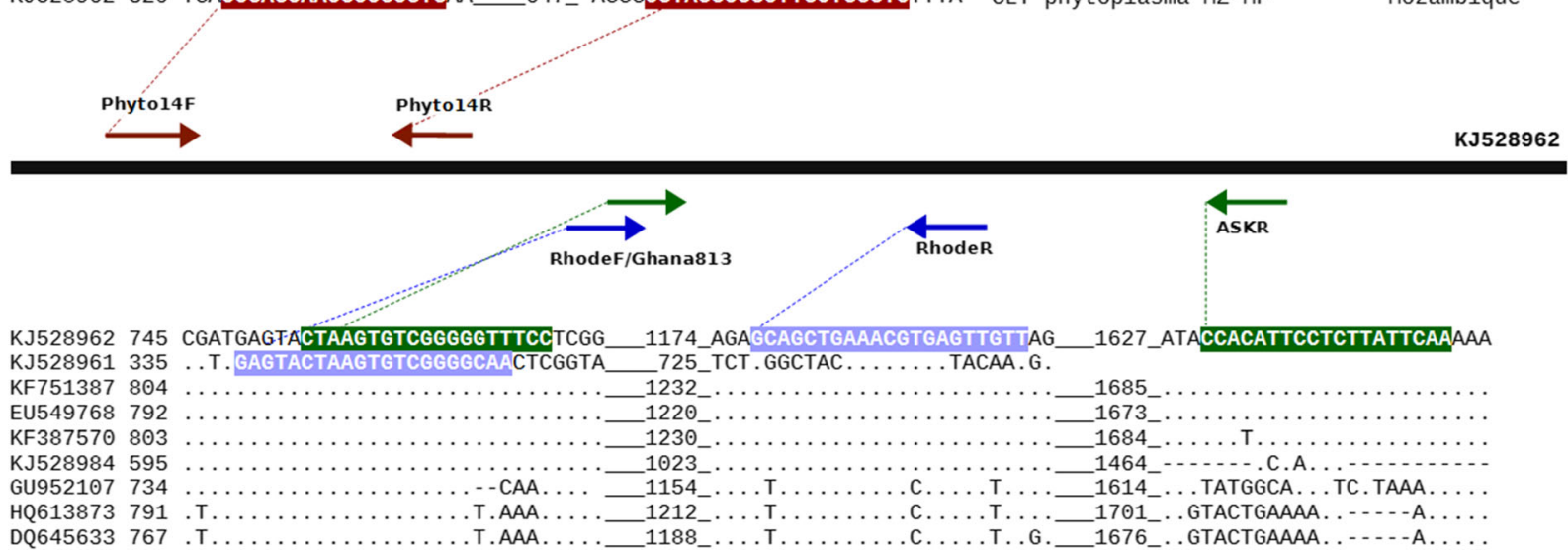

Fig. 1 Diagram showing the relative position of the Phyto14F/R, Ghana813/ASKR and RhodeF/R primer pairs on the 16S rDNA of a CLYD phytoplasma reference strain (Accession no. KJ528962).
Alignment of the annealing regions of 9 representative CLYD phytoplasma published strains is shown 
high genetic variability of these organisms, often reflected in their geographic distribution, may hinder the efficacy of a universal diagnostic method based on the amplification of conserved regions, such as a $16 \mathrm{~S}$ rDNA-based PCR.

Our diagnostic approach based on the PCR was initially designed to use $16 \mathrm{~S}$ universal primers $\mathrm{P} 1 / \mathrm{P} 7$, as well as the other two previously described primers pairs G813/AKSR and RhodeF/R. This procedure worked well until 2012, when clear symptomatic palms started to result positive only to universal primers and not to the regional primers, suggesting a possible genetic diversity of the Mozambican phytoplasma strains under study. Around the same time, Harrison reported that the G813/AKSR primer pair was not able to detect phytoplasma in infected palms and reported the existence of a new variant circulating in Mozambique (Harrison et al. 2014). Recently, Bila et al. (2015) confirmed the presence of two strains of phytoplasma in Mozambique ('Ca. P. palmicola' related strains $16 \mathrm{SrXXII-B}$ and 'Ca. P. pini' related strains), stressing the difficulties of an effective diagnostic by PCR due to the high grade of variability in $16 \mathrm{~S}$ rRNA regions with available primers.

Therefore, in the present study we aimed to develop a diagnostic molecular method able to detect phytoplasma strains infecting coconuts in Mozambique. We designed a very effective primer pair, Phyto14F/R, selected for its consistency to detect phytoplasma strains, not only strains of East and West Africa groups, but also those previously gone undetected in Mozambique. The primer pair shows a significant increase in detection sensitivity compared to the G813/AKSR and RohdeF/R primer pairs.

The use of Phyto14F/R needs to be extended to different countries affected by CLYD to evaluate its diagnostic capability within CLYD control strategies.

Acknowledgments We thank Dr. J. Bila, E. Mondlane University, Mozambique, for his collaboration and advises, Dr. D. Bosco for manuscript revision and Dr. S. Sabatelli, Sapienza University of Rome, Italy, Dr. Massimo Deligios Porto Conte Ricerche, Italy, for technical assistance.

This research was supported by the MCTESTP FNI Grant Number 126-Inv/FNI and by the Italian Agency for Cooperation and Development (AICS) project AID10524.

\section{Compliance with ethical standards}

Conflict of interest The authors declare that they have no conflict of interest.
Open Access This article is distributed under the terms of the Creative Commons Attribution 4.0 International License (http:// creativecommons.org/licenses/by/4.0/), which permits unrestricted use, distribution, and reproduction in any medium, provided you give appropriate credit to the original author(s) and the source, provide a link to the Creative Commons license, and indicate if changes were made.

\section{References}

Abdollahi, F., Niknam, V., Ghanati, F., Masroor, F., \& Noorbakhsh, S. N. (2012). Biological effects of weak electromagnetic field on healthy and infected lime (Citrus aurantifolia) trees with Phytoplasma. The Scientific World Journal, 2012, 716929.

Bai, X., Zhang, J., Ewing, A., Miller, s. a., Radek, A. J., Shevchenko, D. V., Tsukerman, K., Walunas, T., Lapidus, A., Campbell, J. W., \& Hogenhout, S. A. (2006). Living with Genome Instability: the Adaptation of Phytoplasmas to Diverse Environments of Their Insect and Plant Hosts. Journal of Bacteriology, 188(10), 3682-3696.

Bertaccini, A., Duduk, B., Paltrinieri, S., \& Contaldo, N. (2014). Phytoplasmas and Phytoplasma diseases: A severe threat to agriculture. American Journal of Plant Sciences, 1763-1788.

Bila, J., Mondjana, A., Samils, B., Högberg, N. (2014). High diversity, expanding populations and purifying selection in Phytoplasmas causing coconut lethal yellowing in Mozambique. Plant pathology.

Bila, J., Högberg, N., Monjana, A., \& Samils, B. (2015). African fan palm (Borassus aethiopum) and oil palm (Elaeis guineensis) are alternate hosts of coconut lethal yellowing phytoplasma in Mozambique. African Journal of Biotechnology., 14(52), 3359-3367.

Bila, J., Mondjana, A., Samils, B., Högberg, N., Wilson, M. R., \& Santos, L. (2017). First report of 'Candidatus Phytoplasma palmicola' detection in the planthopper Diostrombus mkurangai in Mozambique. Bulletin of Insectology, 70(1), 45-48.

Bonnot, F., De Franqueville, H., \& Lourenço, E. (2010). Spatial and spatiotemporal pattern analysis of coconut lethal yellowing in Mozambique. Phytopathology, 300-312.

Christensen, N. M., Nicolaisen, M., Hansen, M., \& Schulz1, A. (2004). Distribution of Phytoplasmas in Infected Plants as Revealed by Real-Time PCR and Bioimaging. Molecular Plant-Microbe Interactions, Dinamarca., 17(11), 11751184.

Contaldo, N., Bertaccini, A., Paltrinieri, S., Windsor, H. M., \& Windsor, G. D. (2012). Axenic culture of plant pathogenic phytoplasmas. Phytopathologia Mediterranea, 51, 607-617.

Deng, S., \& Hiruki, C. (1991). Amplification 16S rRNA genes from culturable and non-culturable mollicutes. Journal of Microbiological Methods, 14, 53-61.

Dickinson, M. (2010). Micro commentary: Mobile units of DNA in phytoplasma genomes. Molecular Microbiology, 77(6), 1351-1353.

Doyle, J. J., \& Doyle, J. L. (1990). Isolation of plant DNA from fresh tissue. Focus (life technol. Inc.), 12, 13-15. 
Eden-Green, S. J. (2006). Executive summary, excerpts and conclusions of an assessment of coconut lethal yellowing-type disease (LYD) in Mozambique. Millennium Challenge Corporation.

Gundersen, D. E., Lee, I. M., Rehner, S. A., Davis, R. E., \& Kingsbury, D. T. (1994). Phylogeny of Mycoplasmalike organisms (Phytoplasmas): A basis for their classification. American Society for Microbiology, 176(17), 5244-5254.

Harrison, N. A., Davis, R. E., Oropeza, C., Helmick, E. E., Narvaez, M., Eden-Green, S., Dollet, M., \& Dickinson, M. (2014). Candidatus Phytoplasma palmicola', associated with a lethal yellowing-type disease of coconut (Cocos nucifera L.) in Mozambique. International Journal of Systematic and Evolutionary Microbiology, 64, 1890-1899.

Husain, A., Awadhi, A., Hanif, A., Suleman, P., and Montasser, M., (2002). Molecular and microscopical Detection of Phytoplasma Associated with Yellowing Disease of Date Palms Phoenix dactylifera L. In Kuwait. Kuwait J. Sci. Eng, 29(2).

Kearse, M., Moir, R., Wilson, A., Stones-Havas, S., Cheung, M., Sturrock, S., Buxton, S., Cooper, A., Markowitz, S., Duran, C., Thierer, T., Ashton, B., Mentjies, P., \& Drummond, A. (2012). Geneious basic: An integrated and extendable desktop software platform for the organization and analysis of sequence data. Bioinformatics, 28(12), 1647-1649.

Kube, M., Schneider, B., Kuhl, H., Dandekar, T., Heitmann, K., Migdoll, A. M., Reinhard, R., \& Seemüller, E. (2008). The linear chromosome of the plant pathogenic mycoplasma 'Candidatus Phytoplasma Mali'. BMC Genomics, 9, 306.

Larkin, M. A., Blackshields, G., Brown, N. P., Chenna, R., McGettigan, P. A., McWilliam, H., Valentin, F., Wallace, I. M., Wilm, A., Lopez, R., Thompson, J. D., Gibson, T. J., \&
Higgins, D. G. (2007). Clustal W and Clustal X version 2.0. Bioinformatics, 23, 2947-2948.

Lee, I.-M., Davis, R. E., \& Rindal, D. E. G. (2000). Phytoplasma: Phytopathogenic Mollicutes. Revista de Microbiologia, 54, 221-255.

Llauger, R., Becker, D., Cueto, J., Peralta, E., Gonzalez, V., Rodr1'guez, M., \& Rohde, W. (2002). Detection and Molecular Characterization of Phytoplasma associated with Lethal Yellowing Disease of Coconut Palms in Cuba. J. Phytopathology, 150, 390-395.

Monjana, A., Santos, L., Vaz A. Caniço Mangana, S., Nuaila, V., Massingue, F. And Massinga, J. (2010). Manual Prático para Identificação e Maneio do Amarelecimento letal do coqueiro. MTF/MOZ/098/STF (STDF 230)-FAO.

Oropeza, C., Cordova, I., Chumba, A., Narvaez, M., Saenz, L., Ashburner, R., \& Harrison, N. (2011). Phytoplasma distribution in coconut palms affected by lethal yellowing disease. The Annals of Applied Biology, 159, 109-117.

Razin, S. (2007). Molecular biology and genomics of Mollicutes. Bulletin of Insectology, 60(2), 101-103.

Rohde, W., Kullaya, A., Mpunami, A., \& Becker, D. (1993). Rapid and sensitive diagnosis of mycoplasma-like organisms associated with lethal disease of coconut palm by a specifically primed polymerase chain reaction for the amplification of 16SrDNA. Oleagineux, 48, 319-322.

Tymon, A. M., Jones, P., \& Harrison, N. A. (1998). Phylogenetic relationships of coconut phytoplasmas and the development of specific oligonucleotide PCR primer. Annals of Applied Biology, 132, 0437-0452.

Weintraub, P. G., \& Beanland, L. (2006). Insect vectors of phytoplasmas. Annual Review of Entomology, 51, 91-111. 